\title{
Stability of Non-Neutral and Neutral Dynamic Switched Systems Subject to Internal Delays
}

\author{
${ }^{1}$ M. De la Sen, ${ }^{2}$ J.L. Malaina, ${ }^{2}$ A. Gallego and ${ }^{2}$ J.C. Soto \\ Institute of Research and Development of Processes \\ ${ }^{1}$ Department of Electricity and Electronics, Campus of Leioa. Aptdo. 644 de Bilbao \\ ${ }^{2}$ Department of Applied Mathematics, Campus of Bilbao-La Casilla \\ University in Basque Country, Spain
}

\begin{abstract}
This study deals with the quadratic stability and linear state-feedback and output-feedback stabilization of switched delayed linear dynamic systems with, in general, a finite number of non commensurate constant internal point delays. The results are obtained based on Lyapunov's stability analysis via appropriate Krasovsky-Lyapunov's functionals and the related stability study is performed to obtain both delay independent and delay dependent results. It is proved that the stabilizing switching rule is arbitrary if all the switched subsystems are quadratically stable and that it exists a (in general, non-unique) stabilizing switching law when the system is polytopic, stable at some interior point of the polytope but with non-necessarily stable parameterizations at the vertices defining the subsystems.
\end{abstract}

Key words: Asymptotic stability, Quadratic stability, Uniform stability, Convexity problems, Uncommensurate time-delay systems, Neutral time- delay systems

\section{INTRODUCTION}

Switching systems are hybrid dynamical systems composed of subsystems with their own parameterizations subject to a rule orchestrating the switching law between the various subsystems. In the last years, there has been increasing interest in stability and stabilization for switched dynamic systems ${ }^{[1-10]}$ where appropriate switching laws decide through time which subsystem parameterizes the system so that stability is guaranteed. In particular, switching rules are applied $^{[8-10]}$ among several estimation schemes of a given linear plant which are then used to obtain different time-updated parameterizations of the adaptive controller. The switching law orchestrating the various estimators to obtain the active one which parameterizes the adaptive controller is interpreted as a higher hierarchical decision level of the whole adaptive system while the basic adaptation scheme is the lowest decision level. The switching law is designed so that the identification error is minimized in real time while the closed-loop system is guaranteed to be stable. A key motivation for studying switched systems is that many practical systems are inherently multi-model in the sense that several dynamic subsystems describe their whole behavior depending on multiple environmental factors $^{[5]}$. On the other hand, time-delay systems offer an increasing interest since many real - life examples are subject to delays, like, for instance, population growth models, signal transmission, tele-operation problems, wear/peace models and actuator monitored processes with noisy sensors etc., ${ }^{[11]}$. Delays may be classified as point delays or distributed delays according to their nature and as external (i.e. in the inputs or outputs) and internals (i.e., in the state) according to the signals they influence. Point delays may be commensurate if each delay is an integer multiple of a base delay or, more generally, incommensurate if they are arbitrary real numbers ${ }^{[11-14]}$. The presence of internal delays leads to a large complexity in the resulting system's dynamics since the whole dynamical system becomes infinite-dimensional. This fact increases, in addition, the difficulty in the study of basic properties, like for instance controllability, observability, stability and stabilization and robustness, compared to the delay-free case since the transfer functions consist of transcendent numerator and denominator quasi-polynomials ${ }^{[11-38]}$. By those reasons, the design of exact or approximate poleplacement controllers towards the achievement of a finite or infinite closed-loop spectrum becomes of increased difficulty related to the delay-free case ${ }^{[9-18]}$. Neutral delay systems, which are those where the delayed time-derivative influences the system's dynamics ${ }^{[11,28,29]}$, present even a higher analysis and design difficulty. A great effort has been devoted to the investigation of the behavior of time-delay systems in sliding mode and in the use of such a property for synthesizing appropriate controllers ${ }^{[23-32,36-38]}$ including applications to vibrations in heat exchanger tubes and aircraft dynamics ${ }^{[35,36]}$. An important point is that different types of delays appear in a natural way when modeling discrete systems and some classes of hybrid systems $^{[39-41]}$. The objective of this study is to investigate the stability and stabilization properties of linear switched time-delay dynamic systems subject to, in general, multiple incommensurate known internal point delays.

Corresponding Author: M. De la Sen, Institute of Research and Development of Processes, Department of Electricity and Electronics. Campus of Leioa. Aptdo. 644 de Bilbao 


\section{Notation:}

* The sets $\mathbf{R}(Z), \mathbf{R}^{+}\left(\mathbf{Z}^{+}\right)$and $\mathbf{R}_{0}^{+}\left(\mathbf{Z}_{0}^{+}\right)$denote, respectively, the sets of real (integer) numbers, positive real (integer) members and non negative real (integer) numbers.

* It is said that a complex matrix is strictly Hurwitzian, or a stability matrix, if all its eigenvalues have negative real parts.

* An unforced linear system with $\mathrm{r}$ finite incommensurate internal point delays $h_{i}$ of state equation $\dot{x}(t)=A x(t)+\sum_{i=1}^{r} A_{i} x\left(t-h_{i}\right)$ has two associate systems without delays, namely:

$\dot{z}_{1}(t)=\left(A+\sum_{i=1}^{r} A_{i}\right) z_{1}(t)$ Which describes the above so-called current delay-free system time-delay system when $\mathrm{h}_{\mathrm{i}}=0 ; \mathrm{i}=\overline{1, \mathrm{r}} ;$ and $\dot{\mathrm{z}}_{2}(\mathrm{t})=\mathrm{Az}_{2}(\mathrm{t})$ which is called the nominal delay-free system which describes the above time-delay system when $\mathrm{A}_{\mathrm{i}}=0$, or when $\mathrm{h}_{\mathrm{i}} \rightarrow \infty ; \mathrm{i}=\overline{1, \mathrm{r}}$.

Both systems have to be stable in order that the delay system is a stable independent of the delays. The system is said to be g.a.s. if it is globally asymptotically Lyapunov stable and g.u.a.s. If it is globally uniformly asymptotically stable:

* The $l_{2}$-norm of a matrix (or vector) $\mathrm{M}$ is denoted as $\|M\|_{2}=\lambda_{\text {Max }}^{1 / 2}\left(M^{T} M\right)$. In Euclidean vectors, such a norm coincides with the Euclidean norm. A positive definite (semidefinite) matrix $M$ is denoted as $\mathrm{M}>0 \quad(\mathrm{M} \geq 0)$. A negative definite (semidefinite) matrix $\mathrm{M}$ is denoted as $\mathrm{M}<0$ $(\mathrm{M} \leq 0)$.

* The notation for the subset $\{1,2, \ldots, \mathrm{k}\}$ of $\mathbf{Z}^{+}$is abbreviated as $\overline{\mathrm{k}}$.

\section{STABILITY AND STABILIZATION WITH ARBITRARY SWITCHING LAW}

Asymptotic stability independent of and dependent on the delays: Consider the time- varying switched linear dynamic system:

$$
\begin{aligned}
& \Sigma_{\sigma(\mathrm{t})}: \\
& \dot{x}(t)=A_{\sigma(t)} x(t)+\sum_{j=1}^{r} A_{j \sigma(t)} x\left(t-h_{j}\right)+B_{\sigma(t)} u(t) \\
& \mathrm{y}(\mathrm{t})=\mathrm{C}_{\sigma(\mathrm{t})} \mathrm{x}(\mathrm{t})
\end{aligned}
$$

where, $\quad x(t) \in \mathbf{R}^{\mathbf{n}}, \quad u(t) \in \mathbf{R}^{m}, \quad y(t) \in \mathbf{R}^{p}$ are the $\mathrm{n}$ state, $\mathrm{m}$ - input and p-output, respectively, $\mathrm{A}_{\sigma(\mathrm{t})}$, $A_{j \sigma(t)} ; j \in \bar{r}=\{1,2, \ldots, \mathrm{r}\}$, are real square n- matrices describing, respectively, the delay-free dynamics and the various delayed dynamics and $B_{\sigma(t)} \in \mathbf{R}^{m \times n}$ and $C_{\sigma(t)} \in \mathbf{R}^{n \times p}$ are real control and output matrices. The initial condition of (1) is any absolutely continuous function $\varphi:[-h, 0] \rightarrow \mathbf{R}^{\mathbf{n}}$ plus, eventually, a function of zero measure of isolated bounded discontinuities defined on $[-h, 0], \quad$ with $x(0)=\varphi(0)=x_{0}, \quad$ where $h=\underset{1 \leq i \leq r}{\operatorname{Max}}\left(h_{i}\right)$, with $h_{i} \geq 0, \mathrm{i} \in \overline{\mathrm{r}}$ being the delays. The time function $\sigma:[0, \infty) \rightarrow \bar{N}=\{1,2, \ldots, N\}$ is a switching function among the various subsystems $\Sigma_{\mathrm{i}}$ defined at time $\mathrm{t}$ by (1)-(2) for $\sigma(\mathrm{t})=\mathrm{i} \in \bar{N}$ being parameterized with the corresponding matrices $A_{i}, A_{j i} ;, \mathrm{B}_{\mathrm{i}}$ and $\mathrm{C}_{\mathrm{i}} ; \mathrm{j} \in \overline{\mathrm{r}}, i \in \bar{N}$. Thus, $\Sigma_{\sigma(t)} \in\left\{\Sigma_{1}, \Sigma_{2}, \ldots, \Sigma_{N}\right\}$ for all $t \geq 0$.The following set is introduced by convenience:

$S_{i \varepsilon}(\hat{h})=\left\{M_{i}=\left(A_{i}, A_{1 i}, \ldots, A_{r i}\right)\right\} \quad$ such that the switched unforced system $\Sigma_{\mathrm{i}}$ is g.u.a.s. with stability abscissa ; $\left(-\mathcal{E}^{+}\right)$; i.e. with all its eigenvalues satisfying $\operatorname{Max}\left(\operatorname{Re}\left(\lambda_{(.)}\right)\right)=-\varepsilon^{+}<-\varepsilon, \quad$ where $\hat{\mathrm{h}}=\left\{h_{1}, h_{2}, \ldots, h_{r}\right\}$, any $\mathrm{i} \in \bar{N}$. Note, in particular, that $S_{i \varepsilon}(\hat{0})$ is the set of parameterizations of the unforced (1.a) such that the delay- free system $\Sigma_{i}$ is g.u.a.s ; i.e., it is all the set of matrices $\mathrm{A}_{\mathrm{i}}, A_{j i} ; ; j \in \bar{r}, i \in \bar{N}$ such that $\left(A_{i}+\sum_{j=1}^{r} A_{j i}+\mathcal{E} I\right)$ is strictly Hurwitzian. Note also that $S_{i \varepsilon}(\hat{0}) \supset S_{i \varepsilon}(\hat{0}), \quad \forall \varepsilon^{\prime} \in[0, \varepsilon]$. The following result is concerned with arbitrary switching laws while generalizing previous results in $^{[12]}$ to multiple point delays.

Theorem 1: The following items hold:

i. The switched unforced system $\Sigma_{\sigma(t)}$ is g.u.a.s. with quadratic stability independent of the delays for any arbitrary switching law $\sigma .:[0, \infty) \rightarrow \bar{N}$ and $M_{i}=\left(A_{i}, A_{j i}, \ldots, A_{r i}\right) \in S_{i \varepsilon}(\hat{h}), \forall i \in \bar{N}, \forall \hat{h} \in[\hat{0}, \hat{\infty}]$ if there exist $\varepsilon \in \mathbf{R}^{+}$and real matrices $P=P^{T}>0$, $S_{j}=S_{j}^{T}>0, \forall j \in \bar{r}$, such that the following set of matrix constraints holds: 
$Q_{a i}=A_{i}^{T} P+P A_{i}+\sum_{j=1}^{r}\left(S_{j}+P A_{j i} S_{j}^{-1} A_{j i}^{T} P\right)<-\varepsilon I ;$

$\forall \mathrm{i} \in \overline{\mathrm{N}}$

If (3) holds with $\varepsilon \geq 0$ then the switched unforced system $\Sigma_{\sigma(\mathrm{t})}$ is g.a.s. With quadratic stability independent of the delays for any $\sigma:[0, \infty) \rightarrow \overline{\mathrm{N}}$.

ii. The switched unforced system $\Sigma_{\sigma(\mathrm{t})}$ is g.u.a.s. with quadratic stability for any arbitrary switching law $\sigma(\mathrm{t}) \in \overline{\mathrm{N}}, \quad \mathrm{t} \geq 0$ and any delays $\mathrm{h}_{\mathrm{i}} \in\left[0, \mathrm{~h}_{\mathrm{i}}^{0}\right], \forall \mathrm{i} \in \overline{\mathrm{r}}$, if there exist $\varepsilon \in \mathbf{R}^{+}$and real matrices $\mathrm{P}=\mathrm{P}^{\mathrm{T}}>0, \quad \mathrm{~S}_{0 \mathrm{j}}=\mathrm{S}_{0 \mathrm{j}}^{\mathrm{T}}>0$, $\mathrm{S}_{\mathrm{kj}}=\mathrm{S}_{\mathrm{kj}}^{\mathrm{T}}>0, \forall \mathrm{j}, \mathrm{k} \in \overline{\mathrm{r}}$, such that the following set of matrix constraints holds:

$Q_{a i}^{\prime}=\left(A_{i}^{T}+\sum_{j=1}^{r} A_{j i}^{T}\right) P+P\left(A_{i}+\sum_{j=1}^{r} A_{j i}\right)$

$+\sum_{j=0}^{r} \sum_{k=1}^{r} h_{k}^{0} S_{k j}+\sum_{j=1}^{r} h_{j}^{0} P A_{j i} M_{i} R_{j}^{-1} M_{i}^{T} A_{j i}^{T} P<-\varepsilon I$

$\forall \mathrm{i} \in \overline{\mathrm{N}}$

Where:

$M_{i}=\left(A_{i}, A_{j i}, \ldots, A_{r i}\right) ; \forall \mathrm{i} \in \overline{\mathrm{N}}$

$R_{k}=$ Block Diag $\left(S_{k 0}, S_{k 1}, \ldots, S_{k r}\right)$

$; \in \mathbf{R}^{\mathrm{m}(\mathrm{r}+1) \times \mathrm{m}(\mathrm{r}+1)} \forall \mathrm{k} \in \overline{\mathrm{r}}$

If (4) holds with $\varepsilon \geq 0$ then the switched unforced system $\Sigma_{\sigma(\mathrm{t})}$ is g.a.s. with quadratic stability independent of the delays for any $\sigma:[0, \infty) \rightarrow \overline{\mathrm{N}}$ and any delays $\mathrm{h}_{\mathrm{i}} \in\left[0, \mathrm{~h}_{\mathrm{i}}^{0}\right]$, $\forall \mathrm{i} \in \overline{\mathrm{r}}$.

Remark 1: Note that if (3)-(4) hold for some sets of matrices $\mathrm{S}_{\mathrm{kj}}, \mathrm{S}_{\mathrm{kj}}^{(\mathrm{i})}, \mathrm{k}, \mathrm{j} \in \overline{\mathrm{r}}$ then they also hold for some real scalars $\alpha_{j}, \beta_{j}$ with $\alpha_{j}^{2} \geq \beta_{j}^{2}>0$, $\mathrm{j} \in \overline{\mathrm{N}}$. Thus, Theorem 1 (i) holds if:

$$
\begin{aligned}
& A_{i}^{T} P+P A_{i}+\sum_{j=1}^{r}\left(\alpha_{j}^{2} I+\beta_{j}^{-2} P A_{j i} S_{j}^{-1} A_{j i}^{T} P\right) \\
& <-\varepsilon \mathrm{I} ; \forall \mathrm{i} \in \overline{\mathrm{N}} \\
& \left(A_{i}^{T}+\sum_{j=1}^{r} A_{j i}^{T}\right) P+P\left(A_{i}+\sum_{j=1}^{r} A_{j i}\right)
\end{aligned}
$$

$$
\begin{aligned}
& +\sum_{j=0}^{r} \sum_{k=1}^{r} \alpha_{k j}^{2} h_{k}^{0} I+\sum_{j=1}^{r} h_{j}^{0} \beta_{j}^{-2} P A_{j i} M_{i} R_{j}^{-1} M_{i}^{T} A_{j i}^{T} P<-\varepsilon I \\
& ; \forall \mathrm{i} \in \overline{\mathrm{N}}
\end{aligned}
$$

Note that if Theorem 1 (i) holds then Theorem 1 (ii) holds with $\mathrm{h}_{\mathrm{i}}^{0}=0(\mathrm{i} \in \overline{\mathrm{r}})$. Then, the stability depends on the delays may be checked from (7.b) to establish a range of maximum allowable delays.

2.2. Asymptotic stabilization independent of and dependent on the delays: The results of Section 2.1 may be applied to forced stabilizable systems if a stabilizing regulation control law is applied. The discussion is limited to state and output linear feedback. The first definitions are first given.

Definitions 1: The switched unforced system $\Sigma_{\sigma(\mathrm{t})}$ is said to be globally uniformly asymptotically stabilizable (g.u.a.st.) [respectively, globally uniformly asymptotically output stabilizable (g.u.a.o.st.)] with quadratic stability via a linear delay-free control law for any arbitrary switching law $\sigma:[0, \infty) \rightarrow \overline{\mathrm{N}}$ if there is a linear regulation feedback control law $\mathrm{u}_{\sigma(\mathrm{t})}=\mathrm{K}_{\sigma(\mathrm{t})} \mathrm{x}(\mathrm{t})$ with $\mathrm{K}_{\sigma(\mathrm{t})} \in\left\{\mathrm{K}_{1}, \mathrm{~K}_{2}, \ldots, \mathrm{K}_{\mathrm{N}}\right\}$ [respectively, an output regulation feedback control law $\left.\mathrm{u}_{\sigma(\mathrm{t})}=\mathrm{K}_{\sigma(\mathrm{t})}^{0} \mathrm{C}_{\sigma(\mathrm{t})} \mathrm{x}(\mathrm{t})\right]$ for some real matrices $\mathrm{K}_{\mathrm{i}} \in \mathbf{R}^{\mathrm{m} \times \mathrm{n}}$ [respectively, $\quad \mathrm{K}_{\mathrm{i}}^{0} \in \mathbf{R}^{\mathrm{m} \times \mathrm{p}}$ ] $; \forall \mathrm{i} \in \overline{\mathrm{N}}$ such that the closed-loop system:

$\sum_{\sigma(\mathrm{t})}^{\mathrm{c}}:$

$\dot{x}(t)=\left(A_{\sigma(t)}+B_{\sigma(t)} K_{\sigma(t)} C_{\sigma\|t\|}\right) x(t)+\sum_{j=1}^{r} A_{j \sigma(t)} x\left(t-h_{j}\right)$

[respectively:

$\left.\dot{x}(t)=\left(A_{\sigma(t)}+B_{\sigma(t)} K_{\sigma(t)}^{0} C_{\sigma\|t\|}\right) x(t)+\sum_{j=1}^{r} A_{j \sigma(t)} x\left(t-h_{j}\right)\right](8)$

is g.u.a.s. With quadratic stability. The following result whose proof is omitted holds.

Theorem 2: The following items hold:

i. Assume that the switched unforced system $\Sigma \sigma(\mathrm{t})$ is not g.u.a.s. for all delays $h_{i} \in\left[0, h_{i}^{0}\right]$ some $\mathrm{h}_{\mathrm{i}}^{0}>0, \forall \mathrm{i} \in \overline{\mathrm{r}}$, for an arbitrary switching law $\sigma:[0, \infty) \rightarrow \bar{N}$ (i. e. There is a nonempty set $\overline{\mathrm{N}}^{\prime} \subset \overline{\mathrm{N}}$ such that $\mathrm{A}_{\mathrm{i}}$ is not strictly Hurwitzian for $i \in \bar{N}^{\prime}$. Then, a necessary and sufficient condition for the forced $\Sigma_{\sigma(\mathrm{t})}$ to be g.u.a.st. 
(g.u.a.o.st.) for all delays $\mathrm{h}_{\mathrm{i}} \in\left[0, \mathrm{~h}_{\mathrm{i}}^{0}\right]$ some $\mathrm{h}_{\mathrm{i}}^{0}>0, \forall \mathrm{i} \in \overline{\mathrm{r}}$, is that the pair $\left(\mathrm{A}_{\mathrm{i}}, \mathrm{B}_{\mathrm{i}}\right)$ be stabilizable (respectively, the triple $\left(\mathrm{A}_{\mathrm{i}}, \mathrm{B}_{\mathrm{i}}, \mathrm{C}_{\mathrm{i}}\right)$ be stabilizable and detectable) for all $i \in \overline{\mathrm{N}}^{\prime}$. Those conditions guarantee that the switched forced system $\Sigma_{\sigma(t)}$ is g.u.a.st. (respectively, g.u.a.o.st.) independent of the delays provided that $\left\|\mathrm{A}_{\mathrm{ji}}\right\|$; $\mathrm{j} \in \overline{\mathrm{r}}, \mathrm{i} \in \overline{\mathrm{N}}$ is sufficiently small.

ii. Assume that the switched unforced system $\Sigma_{\sigma(\mathrm{t})}$ is g.u.a.s. for arbitrary switching law $\sigma:[0, \infty) \rightarrow \overline{\mathrm{N}}$ for all delays $\mathrm{h}_{\mathrm{i}} \in\left[0, \mathrm{~h}_{\mathrm{i}}^{0}\right]$ some finite $\mathrm{h}_{\mathrm{i}}^{0}>0, \forall \mathrm{i} \in \overline{\mathrm{r}}$. Then there is a (nonunique) linear regulation state-feedback control law $\mathrm{u}_{\sigma(\mathrm{t})}=\mathrm{K}_{\sigma(\mathrm{t})} \mathrm{x}(\mathrm{t}) \quad$ with $\mathrm{K}_{\sigma(\mathrm{t})} \in\left\{\mathrm{K}_{1}, \mathrm{~K}_{2}, \ldots, \mathrm{K}_{\mathrm{r}}\right\}$ for some real matrices $\quad \mathrm{K}_{\mathrm{i}} \in \mathbf{R}^{\mathrm{m} \times \mathrm{n}}$ [respectively, a (nonunique) linear regulated output-feedback control law $\mathrm{u}_{\sigma(\mathrm{t})}=\mathrm{K}_{\sigma(\mathrm{t})}^{0} \mathrm{C}_{\sigma(\mathrm{t})} \mathrm{x}(\mathrm{t}) \quad$ with $\left.\mathrm{K}{ }_{\mathrm{i}}^{0} \in \mathbf{R}^{\mathrm{m} \times \mathrm{p}}\right] ; \forall \mathrm{i} \in \overline{\mathrm{N}}$ such that the resulting closed-loop system $\sum_{\sigma(t)}^{\mathrm{c}}$ is g.u.a.s. with quadratic stability for all delays $\mathrm{h}_{\mathrm{i}} \in\left[0, \mathrm{~h}_{\mathrm{i}} \mathrm{0}^{\prime}\right]$ some finite $\mathrm{h}_{\mathrm{i}}^{0}>\mathrm{h}_{\mathrm{i}}^{0}, \mathrm{i} \in \overline{\mathrm{r}}$, being dependent on the parameterization, if $\left(\mathrm{A}_{\mathrm{i}}, \mathrm{B}_{\mathrm{i}}\right)$ is completely controllable (respectively, the triple $\left(\mathrm{A}_{\mathrm{i}}, \mathrm{B}_{\mathrm{i}}, \mathrm{C}_{\mathrm{i}}\right)$ is completely controllable and observable and $\operatorname{Max}(m, p) \geq n$ ) for all $i \in \bar{N}$.

Definitions 1 may be generalized in a natural way for linear state and output- stabilizability via linear regulation delay- dependent control laws ${ }^{[20-23,32,33]}$. For that purpose, consider the following control laws:

$u(t)=K_{\sigma(t)} x(t)+\sum_{j=1}^{r} K_{j \sigma(t)} x\left(t-h_{j}\right)$

$u(t)=K_{\sigma(t)}^{0} C_{\sigma(t)} x(t)+\sum_{j=1}^{r} K_{j \sigma(t)}^{0} C_{\sigma(t)} x\left(t-h_{j}\right)$

with $\mathrm{K}_{\sigma(\mathrm{t})} \in\left\{\mathrm{K}_{1}, \mathrm{~K}_{2}, \ldots ., \mathrm{K}_{\mathrm{N}}\right\}$,

$\mathrm{K}_{\mathrm{j} \sigma(\mathrm{t})} \in\left\{\mathrm{K}_{\mathrm{j} 1}, \mathrm{~K}_{\mathrm{j} 2}, \ldots ., \mathrm{K}_{\mathrm{jN}}\right\}$,

$\mathrm{K}_{\sigma(\mathrm{t})}^{0} \in\left\{\mathrm{K}_{1}^{0}, \mathrm{~K}_{2}^{0}, \ldots, \mathrm{K}_{\mathrm{N}}^{0}\right\}$

and

$\mathrm{K}_{\mathrm{j} \sigma(\mathrm{t})}^{0} \in\left\{\mathrm{K}_{1 \sigma(\mathrm{t})}^{0}, \mathrm{~K}_{2 \sigma(\mathrm{t})}^{0}, \ldots ., \mathrm{K}_{\mathrm{N} \sigma(\mathrm{t})}^{0}\right\}$ $(\sigma(t) \in \bar{N}, \quad j \in \vec{r})$ for real matrices $K_{i} \in R^{m \times n}$, $\mathrm{K}_{\mathrm{ji}} \in \mathrm{R}^{\mathrm{m} \times \mathrm{n}}, \quad \mathrm{K}_{\mathrm{i}}^{0} \in \mathrm{R}^{\mathrm{m} \times \mathrm{p}} \quad$ and $\quad \mathrm{K}_{\mathrm{ji}}^{0} \in \mathrm{R}^{\mathrm{m} \times \mathrm{p}} ; \mathrm{j} \in \overrightarrow{\mathrm{r}}$, $\mathrm{i} \in \overline{\mathrm{N}}$ specifying the controller gains. Define $\mathrm{n} x \mathrm{n}$ real matrices:

$\hat{\mathrm{A}}_{\mathrm{ji}}=\mathrm{A}_{\mathrm{ji}}+\mathrm{B}_{\mathrm{i}} \mathrm{K}_{\mathrm{i}}$;

$\hat{\mathrm{A}}_{\mathrm{ji}}^{0}=\mathrm{A}_{\mathrm{ji}}+\mathrm{B}_{\mathrm{i}} \mathrm{K}_{\mathrm{i}}^{0} \mathrm{C}_{\mathrm{i}} ; \mathrm{i} \in \overline{\mathrm{N}}$

The following technical result, concerned with the choice of the controller gains corresponding to the delayed dynamics if the control laws (9) or (10), so that the closed-loop delayed dynamics is annihilated or nearly annihilated.

\section{Lemma 1:}

i. If $\left(\mathrm{A}_{\mathrm{ji}}, \mathrm{B}_{\mathrm{i}}\right)$ is a completely controllable pair then it always exists a $\mathrm{K}_{\mathrm{ji}} \in \mathbf{R}^{\mathrm{m} \times \mathrm{n}}$ such that all the zeros of the polynomial $\mathrm{p}_{\mathrm{ji}}(\mathrm{s})=\operatorname{Det}\left(\mathrm{s} I-\mathrm{A}_{\mathrm{ji}}-\mathrm{B}_{\mathrm{i}} \mathrm{K}_{\mathrm{ji}}\right)$ (or, equivalently, $n$ of its $n+1$ coefficients) are located in arbitrary prefixed positions.

ii. If $\left(\mathrm{A}_{\mathrm{ji}}, \mathrm{B}_{\mathrm{i}}, \mathrm{C}_{\mathrm{i}}\right)$ is a completely controllable and observable triple then it always exists a $\mathrm{K} \underset{\mathrm{ji}}{0} \in \mathbf{R}^{\mathrm{m} \times \mathrm{p}}$ such that all the zeros of the polynomial

$\mathrm{p}_{\mathrm{ji}}^{0}(\mathrm{~s})=\operatorname{Det}\left(\mathrm{sI}-\mathrm{A}_{\mathrm{ji}}-\mathrm{B}_{\mathrm{i}} \mathrm{K} \underset{\mathrm{ji}}{0} \mathrm{C}_{\mathrm{i}}\right) \quad$ (or, equivalently, $n$ of its $n+1$ coefficients) are located arbitrarily close to a given set of prefixed positions.

iii. If $\operatorname{rank}\left(B_{i}\right)=m<n$ then for any given real matrix $\hat{\mathrm{A}}_{\mathrm{ji}}^{*}$, it exists a unique $\mathrm{K}_{\mathrm{ji}}=\left(\mathrm{B}_{\mathrm{i}}^{\mathrm{T}} \mathrm{B}_{\mathrm{i}}\right)^{-1} \mathrm{~B}_{\mathrm{i}}^{\mathrm{T}}\left(\hat{\mathrm{A}}_{\mathrm{ji}}^{*}-\mathrm{A}_{\mathrm{ji}}\right)$ gives the minimum value to $\left\|\hat{\mathrm{A}}_{\mathrm{ji}}^{*}-\hat{\mathrm{A}}_{\mathrm{ji}}\left(\mathrm{K}_{\mathrm{ji}}\right)\right\|_{2}^{2}$.

iv. If $\operatorname{rank}\left(B_{i}\right)=n \leq m$ then for any prescribed real matrix $\hat{\mathrm{A}}_{\mathrm{ji}}^{*} \in \mathbf{R}^{\mathrm{n} \times \mathrm{n}}$, it exists a unique $\mathrm{K}_{\mathrm{ji}} \in \mathbf{R}^{\mathrm{m} \times \mathrm{n}}$ such that $\hat{\mathrm{A}}_{\mathrm{ji}}=\hat{\mathrm{A}}_{\mathrm{ji}}^{*}$.

v. If $\operatorname{rank}\left(B_{i}\right)=m$ and $\operatorname{rank}\left(C_{i}\right)=p$ with $\operatorname{Max}(m, p) \geq n$ then for any given real matrix $\hat{\mathrm{A}}_{\mathrm{ji}}^{*}$, it exists a unique matrix:

$\mathrm{K} \underset{\mathrm{ji}}{0}=\left(B_{i}^{T} B_{i}\right)^{-1} B_{i}^{T}\left(\hat{A}_{j i}^{*}-A_{j i}\right) C_{i}^{T}\left(C_{i} C_{i}^{T}\right)^{-1}$

such that gives the minimum value of $\left\|\hat{A}_{\mathrm{ji}}^{*}-\hat{\mathrm{A}}_{\mathrm{ji}}\left(\mathrm{K}_{\mathrm{ji}}^{0}\right)\right\|_{2}^{2}$. 
vi. If

$$
\operatorname{rank}\left(\mathrm{B}_{\mathrm{i}}\right)=\operatorname{rank}\left(\mathrm{C}_{\mathrm{i}}\right)=\mathrm{n} \text { with }
$$

$\operatorname{Max}(\mathrm{m}, \mathrm{p}) \geq \mathrm{n}$ then for any given real matrix $\hat{\mathrm{A}}_{\mathrm{ji}}^{*}$, it exists a unique matrix $\mathrm{K}_{\mathrm{ji}}^{0}=\operatorname{Block} \operatorname{Diag}\left[\mathrm{K}_{\mathrm{ji}}^{0^{*}}, 0\right]$ with $\mathrm{K}_{\mathrm{ji}}^{0^{*} \in \mathbf{R}^{\mathrm{n} \times \mathrm{n}}}$ defined by:

$$
K_{j i}^{0^{*}}=\left(B_{1 i_{i i}}^{T} B_{1 i_{i i}}\right)^{-1}\left(\hat{A}_{j i}^{*}-\hat{A}_{j i}\right)\left(C_{1 i} C_{1 i}^{T}\right)
$$

gives exact matching $\hat{\mathrm{A}}_{\mathrm{ji}}=\hat{\mathrm{A}}_{\mathrm{ji}}^{*}$ (prefixed arbitrarily) where matrix partitions $B_{i}=\left[B_{1 i}, B_{2 i}\right]$ and $\mathrm{C}_{\mathrm{i}}=\left[\mathrm{C}_{1 \mathrm{i}}^{\mathrm{T}}, \mathrm{C}_{2 \mathrm{i}}^{\mathrm{T}}\right]^{\mathrm{T}}$ are used with $\mathrm{B}_{1 \mathrm{i}}, \mathrm{C}_{1 \mathrm{i}}$ being square real n-matrices all $i \in \bar{N}$.

Lemma 1 might be used combined with Theorem 2 in the sense that a control law involving delays allows to reduce the norm of $\hat{\mathrm{A}}_{\mathrm{ji}}$ associated with the delayed dynamics after feedback (defined by one of the two equations in (11) depending on the use of state or output feedback) related to that of $\mathrm{A}_{\mathrm{ji}}$ under the various given conditions of controllability/ observability. This allows to accomplish with the conditions of closed-loop asymptotic stability independent of the delays or to increase the size of the maximum allowable delay guaranteeing closed-loop asymptotic stability via state/output feedback.

\section{ASYMPTOTIC STABILITY AND STABILIZATION WITH A SWITCHING LAW AMONG THE VERTICES OF A POLYTOPIC SYSTEM}

The main result of this section follows below.

Theorem 3: The following two items hold:

i. Assume that there exist square real n-matrices $\mathrm{P}=\mathrm{P}^{\mathrm{T}}>0, \mathrm{~S}_{\mathrm{j}}=\mathrm{S}_{\mathrm{j}}^{\mathrm{T}}>0 \quad(\mathrm{j} \in \overline{\mathrm{r}}), \varepsilon \in \mathbf{R}^{+}$and a $\mathrm{N}$ real scalar $\lambda_{\mathrm{i}} \in(0,1)$ fulfilling $\sum_{\mathrm{k}=1}^{\mathrm{N}} \lambda_{\mathrm{i}}=1$, such that $\overline{\mathrm{Q}}=\sum_{\mathrm{i}=1}^{\mathrm{N}} \lambda_{\mathrm{i}} \mathrm{Q}_{\mathrm{ai}}<0$ with $\mathrm{Q}_{\mathrm{ai}}$ defined in (3), $i \in \bar{N}$. Thus, for any initial condition, there is a (non-unique) switching law $\sigma_{.:}[0, \infty) \rightarrow \overline{\mathrm{N}}$ which is piecewise constant on $[\alpha, \infty)$ for some real constant $\alpha \geq 0$, such that the switched unforced system $\Sigma_{\sigma(\mathrm{t})}$ is g.u.a.s. with quadratic stability independent of the delays. If $\overline{\mathrm{Q}} \geq 0$ for the set of unforced systems $\Sigma_{\mathrm{i}}, \mathrm{i} \in \overline{\mathrm{N}}$, then it always exists a (non-unique) stabilizing switching law $\sigma::[0, \infty) \rightarrow \overline{\mathrm{N}}$ which is piece-wise constant on $[\alpha, \infty)$, some real $\alpha \geq 0$, such that the switched forced system $\Sigma_{\sigma(t)}^{c}$ is g.u.a.s. with quadratic stability independent of the delays provided that any of the conditions below hold:

a. The pair $\left(A_{i}, B_{i}\right)$ is stabilizable for all $i \in \bar{N}$ and a control law $\mathrm{u}(\mathrm{t})=\mathrm{K}_{\sigma(\mathrm{t})} \mathrm{x}(\mathrm{t})$ is generated, with $\mathrm{K}_{\sigma(\mathrm{t})} \in\left\{\mathrm{K}_{1}, \mathrm{~K}_{2}, \ldots, \mathrm{K}_{\mathrm{r}}\right\}$, for controller gains $\mathrm{K}_{\mathrm{i}} \in \mathbf{R}^{\mathrm{m} \times \mathrm{n}}$ is applied.

b. The triple $\left(\mathrm{A}_{\mathrm{i}}, \mathrm{B}_{\mathrm{i}}, \mathrm{C}_{\mathrm{i}}\right)$ is stabilizable / detectable for all $i \in \overline{\mathrm{N}}$ and an output-feedback control law $u(t)=K_{\sigma(t)}^{0} C_{\sigma(t)} x(t)$ is generated, with $\mathrm{K}_{\sigma(\mathrm{t})}^{0} \in\left\{\mathrm{K}_{1}^{0}, \mathrm{~K}_{2}^{0}, \ldots, \mathrm{K}_{\mathrm{r}}^{0}\right\}$, for controller gains $\mathrm{K}_{\mathrm{i}}^{0} \in \mathbf{R}^{\mathrm{m} \times \mathrm{p}}$.

Furthermore, if Error! Bookmark not defined. for the set of unforced systems $\Sigma_{\mathrm{i}}, \mathrm{i} \in \overline{\mathrm{N}}$, but there exists a set of matrices $K_{i} \in \mathbf{R}^{\mathrm{m} \times \mathrm{n}}, i \in \overline{\mathrm{N}}$ and a set of real scalars $\lambda_{i} \in(0,1)$ fulfilling $\sum_{i=1}^{N} \lambda_{i}=1$ such that $\overline{\mathrm{Q}}^{\mathrm{c}}=\sum_{\mathrm{k}=1}^{\mathrm{N}} \lambda_{\mathrm{i}} \mathrm{Q}_{\mathrm{ak}}^{\mathrm{c}}<0$, with $\mathrm{Q}_{\mathrm{ak}}^{\mathrm{c}}$ being redefined from $\mathrm{Q}_{\mathrm{ak}}$ by replacing $\mathrm{A}_{\mathrm{i}} \rightarrow \mathrm{A}_{\mathrm{i}}+\mathrm{B}_{\mathrm{i}} \mathrm{K}_{\mathrm{i}}$ $(i \in \bar{N})$, then the switched closed-loop system $\sum_{\sigma(t)}^{c}$ for the control law $\mathrm{u}(\mathrm{t})=\mathrm{K}_{\sigma(\mathrm{t})} \mathrm{x}(\mathrm{t})$ and some stabilizing switching law $\sigma:[0, \infty) \rightarrow \overline{\mathrm{N}}$ is g.u.a.s. with quadratic stability independent of the delays (even if $\left(A_{i}, B_{i}\right)$ and then $\left(\lambda_{i} A_{i}, \lambda_{i} B_{i}\right)$, is not stabilizable for all $i \in \overline{\mathrm{N}}$ ) .

ii. Assume that there exists square symmetric positive definite matrices $P, S_{0 j}, S_{k j}(k, j \in \bar{r})$ and some real scalars $\lambda_{i} \in(0,1)$ fulfilling $\sum_{k=1}^{N} \lambda_{i}=1$, such that $\overline{\mathrm{Q}}=\sum_{\mathrm{i}=1}^{\mathrm{N}} \lambda_{\mathrm{i}} \mathrm{Q}_{\mathrm{ai}}^{\prime}<0$ with $\mathrm{Q}_{\mathrm{ai}}^{\prime}$ defined in (4)-(5), $\mathrm{i} \in \overline{\mathrm{N}}$. Thus, for any initial conditions, there is a (non-unique) switching law $\sigma:[0, \infty) \rightarrow \overline{\mathrm{N}}$, which is piece-wise constant on $[\alpha, \infty)$ some real $\alpha \geq 0$, such that the switched unforced system $\Sigma_{\sigma(\mathrm{t})}$ is g.u.a.s. with quadratic stability dependent 
of the delays, $\forall \mathrm{h}_{\mathrm{i}} \in\left[0, \mathrm{~h}_{\mathrm{i}}^{0}\right]$, some $\mathrm{h}_{\mathrm{i}}^{0}>0$, all $\mathrm{i} \in \overline{\mathrm{N}}$. If $\overline{\mathrm{Q}} \geq 0$ for the set of unforced systems $\Sigma_{\mathrm{i}}, \quad i \in \overline{\mathrm{N}}$, then it always exists a (non-unique) stabilizing switching law $\sigma:[0, \infty) \rightarrow \overline{\mathrm{N}}$ which is piece-wise constant on $[\alpha, \infty)$ some real $\alpha \geq 0$, such that the switched closed-loop system $\sum_{\sigma(\mathrm{t})}^{\mathrm{c}}$ is g.u.a.s. with quadratic stability dependent of the delays, $\forall \mathrm{h}_{\mathrm{i}} \in\left[0, \mathrm{~h}_{\mathrm{i}}^{0}\right]$, some $\mathrm{h}_{\mathrm{i}}^{0}>0$, all $\mathrm{i} \in \overline{\mathrm{N}}$, provided that similar conditions as (a)-(b) in Item (i) hold. Furthermore, for any prefixed set $\mathrm{h}_{\mathrm{i}}^{0}$ $(i \in \bar{N}), \quad$ it always exist matrices $A_{j i}(j \in \bar{r}, i \in \bar{N})$ and a positive real constant $\gamma$ dependent on $\mathrm{h}_{\mathrm{i}}^{0}(\mathrm{i} \in \overline{\mathrm{N}})$ such that for sufficiently small $\left\|A_{j i}\right\| \leq \gamma \quad(j \in \bar{r}, i \in \bar{N})$, the switched $\Sigma_{\sigma(\mathrm{t})}^{\mathrm{c}}$ obtained under linear state feedback via a controller of gain $\mathrm{K}_{\sigma(\mathrm{t})} \in\left\{\mathrm{K}_{1}, \mathrm{~K}_{2}, \ldots, \mathrm{K}_{\mathrm{r}}\right\}$ is g.u.a.s. with quadratic stability dependent of the delays, $\forall \mathrm{h}_{\mathrm{i}} \in\left[0, \mathrm{~h}_{\mathrm{i}}^{0}\right]$ for some switching law $\sigma:[0, \infty) \rightarrow \overline{\mathrm{N}}$.

Furthermore, if $\overline{\mathrm{Q}} \geq 0$ for the set of unforced systems $\Sigma_{\mathrm{i}}, \mathrm{i} \in \overline{\mathrm{N}}$, but there exists a set of matrices $\mathrm{K}_{\mathrm{i}} \in \mathbf{R}^{\mathrm{m} \times \mathrm{P}}, \quad \mathrm{i} \in \overline{\mathrm{N}}$ and a set of real scalars $\lambda_{i} \in(0,1) \quad$ fulfilling $\quad \sum_{i=1}^{N} \lambda_{i}=1 \quad$ such that $\bar{Q}^{-c}=\sum_{k=1}^{N} \lambda_{i} Q^{\prime c}{ }_{a k}^{c}<0$, with $Q^{\prime c}{ }^{c}$ being redefined from $\mathrm{Q}^{\prime}{ }_{\mathrm{ak}}$ by replacing $\mathrm{A}_{\mathrm{i}} \rightarrow \mathrm{A}_{\mathrm{i}}+\mathrm{B}_{\mathrm{i}} \mathrm{K}_{\mathrm{i}}^{0} \mathrm{C}_{\mathrm{i}}$ $(i \in \bar{N})$, then the switched closed-loop system $\sum_{\sigma(t)}^{c}$ for the control law $u(t)=K{ }_{\sigma(t)}^{0} C_{\sigma(t)} x(t)$ and some stabilizing switching law $\sigma:[0, \infty) \rightarrow \overline{\mathrm{N}}$ is g.u.a.s. with quadratic stability dependent of the delays (even if $\left(A_{i}, B_{i} C_{i}\right)$ and then $\left(\lambda_{i} A_{i}, \lambda_{i} B_{i}, \lambda_{i} C_{i}\right)$, is not stabilizable and detectable for all $i \in \overline{\mathrm{N}}$ ).

An interpretation of Theorem 3 is as follows. The conditions of Theorem 3 (i) imply that if a polytopic system:

$$
\begin{aligned}
& \dot{x}(t)=\sum_{i=1}^{N}\left\{\lambda_{i} A_{i} x(t)+\sum_{j=1}^{r} A_{j i} x\left(t-h_{j}\right)+B_{i} u(t)\right\} ; \\
& \lambda_{i} \in(0,1), \sum_{i=1}^{N} \lambda_{i}=1
\end{aligned}
$$

is g.u.a.s. for zero control input guaranteed by the condition $\sum_{\mathrm{i}=1}^{\mathrm{N}} \lambda_{\mathrm{i}} \mathrm{Q}_{\mathrm{ai}}<0$; i.e., it is g.u.a.s. independent of the delays at some interior point of the polytope being the set defined by some combination of vertices defined by the matrices $Q_{a i}(i \in \bar{N})$ then there is a switching law $\sigma::[0, \infty) \rightarrow \overline{\mathrm{N}}$ such that the corresponding unforced switched $\Sigma_{\sigma(t)}$ is g.u.a.s. The same idea might be extended by switching closed-loop system for the state or output linear feedback under the corresponding modifications given conditions as well as for stability dependent of the delays . That means, roughly speaking, that stability at a point inside the polytope implies stability at any interior point of the polytope (for some switching law) even if the system is not stable at any vertex. Note that $\mathrm{Q}>0 \Leftrightarrow \mathrm{Q}_{\mathrm{a}}<0 \neg \Rightarrow \mathrm{Q}_{\mathrm{i}}>0 \Leftrightarrow \mathrm{Q}_{\mathrm{ai}}<0$ some $\mathrm{i} \in \overline{\mathrm{N}}$ but only $\mathrm{x}^{\mathrm{T}}(\mathrm{t}) \mathrm{Q}_{\mathrm{a}} \mathrm{x}(\mathrm{t})<0 \Rightarrow \mathrm{x}^{\mathrm{T}}(\mathrm{t}) \mathrm{Q}_{\mathrm{ai}} \mathrm{x}(\mathrm{t})<0$ for all nonzero $x(t)$ and all $t \geq 0\left(^{[2,4-6,8]}\right.$ for a delay-free system). It is now interesting to investigate quadratic stability of a switched system composed of two subsystems with an (uncertain) polytopic-type parameterization which are not necessarily stable. Assume that the switched unforced system $\Sigma_{\sigma(t)} \in\left\{\Sigma_{1}, \Sigma_{2}\right\}$ is defined for all $\mathrm{t} \geq 0$ by one of the two subsequent subsystems:

$$
\Sigma_{i}: \dot{x}(t)=A_{i} x(t)+\sum_{j=1}^{r} A_{j i} x\left(t-h_{j}\right) ; i=1,2
$$

Assume that $\Sigma_{\mathrm{i}}(\mathrm{i}=1,2)$ are uncertain polytopic systems defined by:

$$
A_{i}=\sum_{k=1}^{N_{i}} \mu_{i k} A_{i}^{(k)} ; A_{j i}=\sum_{k=1}^{N_{i}} \mu_{i k} A_{j i}^{(k)}(j \in \bar{r})
$$

with scalars $\mu_{\mathrm{ik}} \in(0,1) ; \mathrm{i}, \mathrm{k}=1,2$ subject to $\sum_{\mathrm{k}=1}^{2} \mu_{\mathrm{ik}}=1 ; \mathrm{i}=1,2 ;$ and real square n-matrices $A_{j i}^{(k)}$ and $A_{j i}^{(k)} ; \mathrm{i}, \mathrm{k}=1,2 ; \mathrm{j} \in \overline{\mathrm{r}}$ defining the delay-free and delayed dynamics at the vertices. For simplicity of exposition and mathematical proofs, it is assumed in the sequel without loss of generality that the number of extreme points $\mathrm{N}_{\mathrm{i}}=2 ; \mathrm{i}=1,2$. In order to make the subsequent discussion nontrivial, the following assumption is made.

Assumption 1: Both $\Sigma_{\mathrm{i}}, \mathrm{i}=1,2$ are not quadratically stable $\forall h_{i} \in\left[0, h_{i}^{0}\right]$, some $h_{i}^{0}>0$, all $i=1,2$; i.e. 
there does not exist real square symmetric n-matrices $\mathrm{P}_{\mathrm{i}}>0(\mathrm{i}=1,2)$ such that:

$$
\left(A_{i}^{(k)}+\sum_{j=1}^{r} A_{j i}^{(k)}{ }^{T} P_{i}+P_{i}\left(A_{i}^{(k)}+\sum_{j=1}^{r} A_{j i}^{(k)}\right)<0 ;\right.
$$$$
\mathrm{i}, \mathrm{k}=1,2
$$

The matrix inequality (17) holds when for each $\mathrm{i}=1,2$, there it at least one matrix $\left(\mathrm{A}_{\mathrm{i}}^{(\mathrm{k})}+\sum_{\mathrm{j}=1}^{\mathrm{r}} \mathrm{A}_{\mathrm{ji}}^{(\mathrm{k})}\right)$ for $\mathrm{k}=1$ or 2 which is not strictly Hurwitzian so that the polytopic system $\Sigma_{i}$ is not quadratically stable for $\mathrm{i}=1,2$ at the corresponding vertex for zero delays. By continuity of the characteristic roots, there is some neighborhood of values of delays around zero such that the corresponding polytopic system is not quadratically stable ; i.e. there exist $\mathrm{h}_{\mathrm{j}}^{0}$ such that $\Sigma_{\mathrm{i}}(\mathrm{i}=1,2)$ is not quadratically stable for all $h_{i} \in\left[0, h_{i}^{0}\right]$. Note that if Assumption 1 holds then Theorem 1 cannot be applied because of the instability or critical stability at the vertices. The following result, whose proof is omitted, is related to the stabilization of (15) via switching.

Theorem 4: The following two items hold under Assumption 1:

i. The switched system $\Sigma_{\sigma(\mathrm{t})}$ is quadratically stable independent of the delays, via some non-unique switching law $\sigma:[0, \infty) \rightarrow \overline{2}=\{1,2\}$, if there exist constant real scalars $\varepsilon>0, \lambda_{\mathrm{ik}} \in(0,1)$; , $\mathrm{k}=1,2 ; \sum_{\mathrm{k}=1}^{2} \lambda_{\mathrm{ik}}=1 ; \mathrm{i}=1,2$ and real square $\mathrm{n}$ matrices $\mathrm{P}=\mathrm{P}^{\mathrm{T}}>0, \quad \mathrm{~S}_{\mathrm{j}}=\mathrm{S}_{\mathrm{j}}^{\mathrm{T}}>0 \quad(\mathrm{j} \in \overline{\mathrm{r}})$ such that:

$$
\begin{aligned}
& {\left[\lambda_{i j} A_{1}^{(i)}+\left(1-\lambda_{i j}\right) A_{2}^{(j)}\right]^{T}} \\
& P+P\left[\lambda_{i j} A_{1}^{(i)}+\left(1-\lambda_{i j}\right) A_{2}^{(j)}\right] \\
& +\left[\begin{array}{l}
\lambda_{i j}\left(\sum_{k=1}^{r} S_{k}+P A_{k 1}^{(i)} S_{k}^{-1} A_{k 1}^{(i)} T^{T} P\right) \\
\left.+\left(1-\lambda_{i j}\right)\left(\sum_{k=1}^{r} S_{k}+P A_{k 2}^{(i)} S_{k}^{-1} A_{k 2}^{(i)}{ }^{T} P\right)\right]<-\varepsilon I
\end{array}\right.
\end{aligned}
$$$$
\text { ; i , j }=1,2
$$

ii. The switched system $\Sigma_{\sigma(\mathrm{t})}$ is quadratically stable for all delays $h_{i} \in\left[0, h_{i}^{0}\right]$, some $h_{i}^{0}>0 \quad(i \in \bar{r})$ via some switching law $\sigma:[0, \infty) \rightarrow \overline{2}$, if there exist constant real scalars $\varepsilon>0, \lambda_{\mathrm{ik}} \in(0,1)$; , $\mathrm{k}=1,2 ; \sum_{\mathrm{k}=1}^{2} \lambda_{\mathrm{ik}}=1 ; \mathrm{i}=1,2$ and real square $\mathrm{n}$ matrices $\quad \mathrm{P}=\mathrm{P}^{\mathrm{T}}>0, \quad \mathrm{~S}_{\mathrm{kj}}=\mathrm{S}_{\mathrm{kj}}^{\mathrm{T}}>0$, $\mathrm{S}_{\mathrm{k} 0}=\mathrm{S}_{\mathrm{k} 0}^{\mathrm{T}}>0(\mathrm{k}, \mathrm{j} \in \overline{\mathrm{r}})$ such that :

$$
\begin{aligned}
& {\left[\lambda_{i j}\left(A_{1}^{(i)}+\sum_{k=1}^{r} A_{k 1}^{(i)}\right)+\left(1-\lambda_{i j}\right)\left(A_{2}^{(i)}+\sum_{k=1}^{r} A_{k 2}^{(i)}\right)\right]{ }^{T} P} \\
& +P\left[\lambda_{i j}\left(A_{1}^{(i)}+\sum_{k=1}^{r} A_{k 1}^{(i)}\right)+\left(1-\lambda_{i j}\right)\left(A_{2}^{(i)}+\sum_{k=1}^{r} A_{k 2}^{(i)}\right)\right] \\
& +\sum_{j=0}^{r} \sum_{k=1}^{r} h_{k}^{0} S_{k j}+ \\
& \lambda_{i j}\left(\sum_{k=1}^{r} h_{k}^{0} P A_{k 1}^{(i)} M_{1}^{(i)} R_{k}^{-1} M_{1}^{(i)} A_{k 1}^{(i) T} P\right) \\
& +\left(1-\lambda_{i j}\right)\left(\sum_{k=1}^{r} h_{k}^{0} P A_{k 2}^{(i)} M_{2}^{(i)} R_{k}^{-1} M_{2}^{(i) T} A_{k 2}^{(i) T} P\right)<-\varepsilon I
\end{aligned}
$$$$
; \mathrm{i}, \mathrm{j}=1,2
$$

\section{ASYMPTOTIC STABILITY OF A CLASS OF UNFORCED NEUTRAL SYSTEMS}

A standard class of unforced neutral systems involving a single point delay is now focused on ${ }^{[11,28]}$. The extensions in the cases of multiple point delays and regulating inputs are direct by using direct extensions with the tools of this class of systems. They are omitted by the sake of simplicity. Consider the neutral system:

$$
\begin{aligned}
& \Sigma_{\sigma(t)}^{\prime}: \dot{x}(t)=A_{\sigma(t)} x(t)+A_{1 \sigma(t)} \\
& x(t-h)+D_{\sigma(t)} \dot{x}(t-h) \\
& =\tilde{A}_{\sigma(t)} x(t)+\tilde{A}_{1 \sigma(t)} x(t-h)+D_{\sigma(t)} \\
& \dot{x}(t-h)-W_{\sigma(t)} \int_{t-h}^{t} \dot{x}(\tau) d \tau
\end{aligned}
$$

where, the function of initial conditions is any absolutely continuous function $\varphi:[-\mathrm{h}, 0] \rightarrow \mathbf{R}^{\mathbf{n}}$ plus, eventually, a function of zero measure of isolated bounded discontinuities defined on $[-\mathrm{h}, 0]$, with $x(0)=\phi(0)=x_{0}$ and $W_{\sigma(t)}$ is chosen so that $\tilde{\mathrm{A}}_{\sigma(\mathrm{t})}=\mathrm{A}_{\sigma(\mathrm{t})}+\mathrm{W}_{\sigma(\mathrm{t})}$ is strictly Hurwitzian for all $\sigma(\mathrm{t}) \in \overline{\mathrm{N}}$ and $\quad \tilde{\mathrm{A}}_{1 \sigma(\mathrm{t})}=\mathrm{A}_{1 \sigma(\mathrm{t})}-\mathrm{W}_{\sigma(\mathrm{t}) \quad \text { with }}$ $\sigma:[0, \infty) \rightarrow \overline{\mathrm{N}}$ being the switching law. All the matrices of parameters in (20) are square real of order $n$ with:

$\mathrm{A}_{\sigma(\mathrm{t})} \in\left\{\mathrm{A}_{1}, \mathrm{~A}_{2}, \ldots, \mathrm{A}_{\mathrm{N}}\right\}$ 


$$
\begin{aligned}
& \mathrm{A}_{1 \sigma(\mathrm{t})} \in\left\{\mathrm{A}_{11}, \mathrm{~A}_{12}, \ldots, \mathrm{A}_{1 \mathrm{~N}}\right\} \\
& \mathrm{D}_{\sigma(\mathrm{t})} \in\left\{\mathrm{D}_{1}, \mathrm{D}_{2}, \ldots, \mathrm{D}_{\mathrm{N}}\right\} \\
& \mathrm{W}_{\sigma(\mathrm{t})} \in\left\{\mathrm{W}_{1}, \mathrm{~W}_{2}, \ldots, \mathrm{W}_{\mathrm{N}}\right\}
\end{aligned}
$$

for all $t \geq 0$. Since $\tilde{A}_{\sigma(t)}$ is strictly Hurwitzian for all $t \geq 0,\left(A_{i}+W_{i}\right)$ is strictly Hurwitzian for all $i \in \bar{N}$. The following result is related to the stability of (20) via switching:

Theorem 5: The neutral system $\Sigma_{\sigma(t)}^{\prime}$ is g.u.a.s. for all delays $h \in\left[0, h^{0}\right]$ and some $h^{0}>0$ for any arbitrary switching law $\sigma::[0, \infty) \rightarrow \overline{\mathrm{N}}$, if $\left\|\mathrm{D}_{\mathrm{i}}\right\|<1$ and $\left(\mathrm{A}_{\mathrm{i}}+\mathrm{W}_{\mathrm{i}}\right)$ is strictly Hurwitzian $(\mathrm{i} \in \overline{\mathrm{N}})$, provided that there exist square real positive definite symmetric nmatrices $\mathrm{P}, \mathrm{S}, \mathrm{R}$ and $\mathrm{T}$ such that:

$$
\begin{aligned}
& \Pi_{\mathrm{i}}=\operatorname{Block} \text { Matrix }\left(\Pi_{\mathrm{ij}} ; \mathrm{i}, \mathrm{j}=1,3\right)<0 \\
& \Pi_{11 i}=\left(A_{i}+W_{i}\right)^{T} P+P\left(A_{i}+W_{i}\right)+ \\
& R+A_{i}^{T}\left(S+h^{0} T\right) A_{i}+h^{0} P W_{i} T^{-1} W_{i}^{T} P \\
& \Pi_{12 i}=\Pi_{21 i}^{T}=P\left(A_{1 i}-W_{i}\right)+A_{i}^{T}\left(S+h^{0} T\right) A_{1 i} ; \\
& \Pi_{13 i}=\Pi_{31 i}^{T}=\left[P+A_{i}^{T}\left(S+h^{0} T\right) D_{i}\right] \text { all } \mathrm{i} \in \overline{\mathrm{N}}
\end{aligned}
$$

The proof of the subsequent result follows immediately from Theorem 5 , via Schur's complement of the last block matrix of $\Pi_{i}=\operatorname{Block}$ Matrix $\left(\Pi_{i j} ; i, j=1,3\right)<0$, in the equivalent test for negative definite ${ }^{[43]}$.

Corollary 1: Theorem 5 holds if:

$$
\begin{aligned}
& \Pi_{11 i}=\operatorname{Block} \text { Matrix }\left(\Pi_{i j} ; i, j=2,3\right)<0 \\
& \left(A_{i}+W_{i}\right)^{T} P+P\left(A_{i}+W_{i}\right)+R+A_{i}^{T}\left(S+h^{0} T\right) A_{i} \\
& -\left(M_{12 i}, M_{13 i}\right)\left(\Pi_{11 i}^{\prime}\right)^{-1}\left(M_{12 i}, M_{13 i}\right)^{T}<0 \\
& \text { all } i \in \bar{N}
\end{aligned}
$$

Now, the polytopic structures of Theorems 4-5 are extended for the given class of switching laws which conditionally stabilize the switched system under convexity-type constraints. The extensions of all the results in this section to the case of forced systems are directly obtained by using linear state/output feedback laws under either controllability/observability or stabilizability/detectability assumptions of the appropriate parameterizations of the subsystems. Some stability results for the neutral system of this section based on the properties of its subsystems are summarized in the following result.
Theorem 6: The following items hold:

i. The neural system $\Sigma_{\sigma(t)}^{\prime}$ is g.u.a.s. for all delays $\mathrm{h} \in\left[0, \mathrm{~h}^{0}\right]$ and some $\mathrm{h}^{0}>0$ for some (nonunique) switching law $\sigma:[0, \infty) \rightarrow \overline{\mathrm{N}}$, if $\left\|\mathrm{D}_{\mathrm{i}}\right\|<1$ and $\left(\mathrm{A}_{\mathrm{i}}+\mathrm{W}_{\mathrm{i}}\right)$ is strictly Hurwitzian $(i \in \bar{N})$, provided that there exist square real symmetric n-matrices $\mathrm{P}, \mathrm{S}, \mathrm{R}$ and $\mathrm{T}$ and some set of real scalars $\overline{\mathrm{N}} \ni \lambda_{\mathrm{i}} \geq 0$ satisfying $\sum_{\mathrm{i}=1}^{\mathrm{N}} \lambda_{\mathrm{i}}=1$, such that $\Pi=\sum_{\mathrm{i}=1}^{\mathrm{N}} \lambda_{\mathrm{i}} \Pi_{\mathrm{i}}<0$, with the matrices $\Pi_{\mathrm{i}}(\mathrm{i} \in \overline{\mathrm{N}})$ being defined in (22)-(23).

ii. Assume that $\Sigma_{\sigma(\mathrm{t})}^{\prime} \in\left\{\Sigma_{1}^{\prime}, \Sigma_{2}^{\prime}\right\}$ for any switching law $\sigma:[0, \infty) \rightarrow \overline{2}$ and all time $\mathrm{t} \geq 0$ where $\Sigma_{\mathrm{i}}^{\prime}$, being defined by (20) when $\sigma(\mathrm{t})$ $=\mathrm{i} \in \overline{2}=\{1,2\}$, are not quadratically stable with $\tilde{\mathrm{A}}_{\mathrm{i}}=\mathrm{A}_{\mathrm{i}}+\mathrm{W}_{\mathrm{i}}$ is strictly Hurwitzian, $\tilde{\mathrm{A}}_{1 \mathrm{i}}=\mathrm{A}_{\mathrm{li}}-\mathrm{W}_{\mathrm{i}}$ and $\left\|\mathrm{D}_{\mathrm{i}}\right\|<1$ for $\mathrm{i}=1,2$. Assume also that $\Sigma_{\mathrm{i}}^{\prime}(\mathrm{i}=1,2)$ are uncertain polytopic systems defined by:

$$
\begin{aligned}
& \mathrm{A}_{\mathrm{i}}=\sum_{\mathrm{k}=1}^{2} \mu_{\mathrm{ik}} \mathrm{A}_{\mathrm{i}}^{(\mathrm{k})} ; \mathrm{A}_{\mathrm{li}}=\sum_{\mathrm{k}=1}^{2_{\mathrm{i}}} \mu_{\mathrm{ik}} \mathrm{A}_{\mathrm{li}}^{(\mathrm{k})}(\mathrm{j} \in \overline{\mathrm{r}}) \\
& \mathrm{W}_{\mathrm{i}}=\sum_{\mathrm{k}=1}^{2} \mu_{\mathrm{ik}} \mathrm{W}_{\mathrm{i}}^{(\mathrm{k})} ; \mathrm{D}_{\mathrm{ji}}=\sum_{\mathrm{k}=1}^{2_{\mathrm{i}}} \mu_{\mathrm{ik}} \mathrm{D}_{\mathrm{i}}^{(\mathrm{k})}(\mathrm{j} \in \overline{\mathrm{r}})
\end{aligned}
$$

via real scalars $\mu_{\mathrm{ik}} \in(0,1) ; \mathrm{i}, \mathrm{k}=1,2$ subject to $\sum_{\mathrm{k}=1}^{2} \mu_{\mathrm{ik}}=1 ; \quad \mathrm{i}=1,2$. Then, there is a non-unique switching law $\sigma:[0, \infty) \rightarrow \overline{2}$ such that $\Sigma_{\sigma(\mathrm{t})}^{\prime}$ is g.u.a.s. for all delays $\mathrm{h} \in\left[0, \mathrm{~h}^{0}\right]$ and some $\mathrm{h}^{0}>0$ provided that the four subsequent linear matrix constraints hold for some square real symmetric nmatrices $\mathrm{P}, \mathrm{S}, \mathrm{R}$ and $\mathrm{T}$ and some set of real scalars $\overline{\mathrm{N}} \ni \lambda_{\mathrm{ik}}>0$ satisfying $\sum_{\mathrm{k}=1}^{2} \lambda_{\mathrm{ik}}=1 ; \mathrm{i}=1,2$ :

$\Pi(i, j)=\lambda_{i j} \Pi_{1}^{(i)}+\left(1-\lambda_{i j}\right) \Pi_{2}^{(j)}<0 ; \mathrm{i}, \mathrm{j}=1,2$,

where $\Pi_{\mathrm{i}}^{(\mathrm{j})}$ is defined similarly as $\Pi_{\mathrm{i}}$ in (21)-(22) with the following replacements related to Theorem 5:

$$
\begin{aligned}
& \left(A_{i}, W_{i}, A_{1 i}, D_{i}\right) \rightarrow \\
& \left(A_{i}^{(j)}, W_{i}^{(j)}, A_{1 i}^{(j)}, D_{i}^{(j)}\right) \quad ; i=1,2
\end{aligned}
$$


Note that if all the parameterizations $\Sigma_{i}{ }^{\prime}$ are not stable then $\lambda_{i} \in(0,1)$ for all $i \in \bar{N}$ in Theorem 1 (i) since $\Pi=\Pi_{k}<0$ is impossible if $\lambda_{k}=1, \lambda_{i}=0$ for some $\mathrm{k} \neq \mathrm{i} \in \overline{\mathrm{N}}$. For Theorem 6(ii) the above constraint has to hold as well by the same reasons that those pointed out related to Theorem 4. The extension of Theorem 6 (ii) to general parameterizations defined by (25) with $\mathrm{N}_{\mathrm{i}} \geq 3 ; \mathrm{i}=1,2$ is direct by using more constraint (26) by involving the corresponding necessary $2^{\mathrm{N}_{\mathrm{i}}}$ constraints in the same way as Theorem 4 is extendable to this situation. The extension is omitted for the sake of simplicity.

\section{CONCLUSION}

This study has been devoted to investigate the stability and stabilization properties of linear switched time-delay dynamic systems being subject to, in general, multiple incommensurate known internal point delays. Firstly, the uniform asymptotic quadratic Lyapunov stability (both independent of and dependent on the delays) for unforced systems has been investigated under arbitrary switching laws in nonpolytopic systems parameterized by a finite set of stable subsystems. The results have been extended to prove the existence of stabilizing switching laws in polytopic systems under testable convexity-type conditions for the vertices. Further stability results have been derived from forced systems for linear state/output feedback control laws under certain controllability and observability/stabilizability and detectability conditions. The stability results have been also extended to a class of switched polytopic systems and two switched systems consisting of a set of polytopic subsystems, subject to mutual switchings through time, which fulfill a convexity-type condition by each combination of sets of vertices, one corresponding to each polytope. Numerical simulated examples have corroborated some of the obtained results.

\section{ACKNOWLEDGMENTS}

The authors are very grateful to MEC and UPV/EHU by their partial support of this work through Grants DPI 2003-00164 and 9/UPV.I06.I06$15263 / 2003$, respectively. They are also grateful to Mr. Ibeas for his interesting comments about the subject.

\section{REFERENCES}

1. Narendra, K.S. and V. Balakrihsnan, 1994. A common Lyapunov function for stable LTI systems with commuting A-matrices. IEEE Trans. Automat. Contr., 39: 774-753.
2. Wicks, M., P. Peleties and R. DeCarlo, 1998. Switched controller synthesis for the quadratic stabilization of a pair of unstable linear systems. Eur. J. Control, 4: 140-147.

3. Xu, X. and P.J. Antsaklis, 2004. Stabilization of second-order LTI switched systems. Intl. J. Control, 73: 1261-1279.

4. Sun, Z. and S.S. Ge, 2003. Dynamic output feedback stabilization of a class of switched linear systems. IEEE Trans. On Circuits and Systems IFundamental Theory and Application, 50: 11111115.

5. Zhai, G., H. Lin and P. Antsaklis, 2003. Quadratic stabilizability of switched linear systems with polytopic uncertainties. Intl. J. Control, March 2004, 76: 2469-2471.

6. Sun, Z., 2004. A robust stabilizing law for switched linear systems. Int. J. Control, 77: 389-398.

7. Wang, F. and V. Balakrishnan, 2002. Improved stability analysis and gain-scheduled controller synthesis for parameter-dependent systems. IEEE Trans. Automate. Contr., 47: 720-734.

8. Bacciotti, A., 2004. Stabilization by means of state space depending switching rules. Systems and Control Lett., 53: 195-201.

9. Alonso-Quesada, S. and M.D.L. Sen, 2004. Robust adaptive control with multiple estimation models for stabilization of a class of non-inversely stable time-varying plants. Asian J. Control, 6: 59-73.

10. Ibeas, A., M. De la Sen and S. Alonso- Quesada, 2004. Stable multi-estimation model for singleinput single-output discrete adaptive control systems. Int. J. Systems Sci., 35: 479-501.

11. Niculescu, S.I., 2001. Delay Effects on Stability. A Robust Control Approach. Lecture Notes in Control and Information, series No. 269 (M. Thoma and M. Morari Eds.), Springer-Verlag, Berlin.

12. Xie, G. and L. Wang, 2004. Stability and stabilization of switched linear systems with state delay: continuous- time case. 6th Intl. Symp. In Mathematical Theory of Networks and Systems, Katholieke Universitat Leuven, Belgium, MTNS 2004, Leuven, July 5-9, Session Switched Linear Systems I, MA2.

13. Jugo, J. and M. de la Sen, 2002. Input-output based pole-placement in a class of time-delay systems. IEE Proc. Control Theory and Appl., 149: 323-330.

14. De la Sen, M., 2004. On pole-placement controllers for linear time-delay systems with commensurate delays", Mathematical Problems in Engineering (in press, 2004).

15. De la Sen, M., 2000. Allocation of poles of delayed systems reduced to those associated with their undelayed counterparts. Elect. Lett., 36: 373-374.

16. De la Sen, M., 2003. On the asymptotic hyperstability of dynamic systems with point delays. IEEE Trans. Circ. Syst. I- Fundamental Theory Applications, 50: 1486-1488. 
17. Galkowski, K., 2001. State-space realizations of linear 2-D systems with extensions to the general $\mathrm{nD}(\mathrm{n}>2$ ) case. Lecture Notes in Control and Information Series, Vol. 263, $1+$.

18. Richard, J.P., 2003. Time-delay systems: an overview of some recent advances and open problems. Automatic, 38: 1667-1694.

19. Yu, Y., 1983. On stabilizing uncertain linear delay systems. J. Optimization Theory and Applications, 41: 503-508.

20. Mahmoud, M.S. and N.F. Almuthairi, 1994. Quadratic stabilization of continuous-time systems with state-delay and norm-bounded time-varying uncertainties. IEEE Trans. Automate. Contr., 39: 2135-2139.

21. Choi, H.H. and M.J. Chung, 1995. Memoryless stabilization of uncertain dynamic- systems with time-varying delayed states and controls. Automatic, 31: 1349-1351.

22. Li, X. and C.E. deSouza, 1997. Criteria for robust stability and stabilization of uncertain linear systems with state delay. Automatic, 33: 16571662.

23. Luo, N.S., M. De la Sen and J. Rodellar, 1997. Robust stabilization of a class of uncertain time delay systems in sliding mode. Int. J. Robust and Nonlinear Control, 76: 59-74.

24. El-Khazali, R., 1998. Variable structure robust control of uncertain time-delay systems. Automatic, 34: 327-332.

25. Richard, J.P., F. Gouaisbaut and W. Perruquetti, 2001. Sliding mode control in the presence of delay", Kybernetika. 37: 277-294.

26. Orlov, Y., W. Perruquetti and J.P. Richard, 2003. Sliding mode control synthesis of uncertain timedelay systems. Asian J. Control, 5: 568-577.

27. Yan, J.J., 2003. Sliding mode control design for uncertain time-delay systems subjected to a class of nonlinear inputs. Intl. J. Nonlinear and Robust Control, 13: 519-532.

28. Chen, J.J., 2004. New stability criteria for a class of neutral systems with discrete and distributed timedelays: an LMI approach. Applied Mathematics and Computation, 150: 719-736.

29. Niu, Y., J. Lam and X. Wang, 2004. Sliding-mode control for uncertain neutral delay systems. IEE Proc. Control Theory and App., 151: 38-44.

30. Niu, Y., J. Lam, X. Wang and D.W.C. Ho, 2004. Observer-based sliding mode control of nonlinear state-delayed systems. Intl. J. Systems Sci., 35: 139-150.

31. Hsu, K.C., 1999. Decentralized sliding mode control of large-scale time-delayed systems with series nonlinearities. J. Dynamic Systems, Measurement and Control- Trans. Of ASME, 121: 708-713.
32. Oucheriah, S., 2003. Exponential stabilization of linear delayed systems using sliding- mode controllers. IEEE Trans. Automate. Contr., 50: 826-830.

33. De la Sen, M., 2004. Sufficiency-type stability and stabilization criteria for linear time-invariant systems with constant point delay. Acta Applicandae Mathematicae, 83: 235-256.

34. De la Sen, M. and N.S. Luo, 2004. On the uniform exponential stability of a wide class of linear timedelay systems. J. Mathematical Analysis and Application, 289: 456-476.

35. De Bedout, J.M., M.A. Franchek and A.K. Bajaj, 1999. Robust control of chaotic vibrations for impacting heat exchanger tubes in cross-flow. J. Sound and Vibration, 227: 183-204.

36. Jafarov, E.M. and R. Tasaltin, 2003. Robust sliding- mode control for the uncertain MIMO aircraft model F-18. IEEE Trans. Aerospace and Electronic Systems, 36: 1127-1141 (2000). IEEE Trans. Automate. Contr., 50: 826-830.

37. Zheng, F. and P.M. Frank, 2000. Finitedimensional variable structure control design for systems with a single time delay. IEEE Trans. Aerospace and Electronic Systems, 36: 1127-1141.

38. Zheng, F. and P.M. Frank, 2001. Finitedimensional variable structure control design for distributed delay systems. Intl. J. Control, 74: 398408.

39. De la Sen, M., 1997. On the hyperstability of a class of hybrid systems. Int. J. Systems Sci., 28: 925-934.

40. Barcena, R., I. Sagastabeitia and M. De la Sen, 2000. Improving the stability properties of the zeros of sampled data systems with fractional order hold. Proc. IEEE Control Theory and Appl., 147: 456-464.

41. Liang, S., M. Ishitobi and Q. Zhu, 2003. Improvement of stability of zeros in discrete-time multivariable systems using fractional - order hold. Int. J. Control, 76: 1699-1711.

42. Kreindle, E. and A. Jameson, 1972. Conditions for nonnegativeness of partitioned matrices. IEEE Trans. Automate. Contr., 17: 147.

43. Zhan, X., 2001. Matrix Inequalities, Lecture Notes in Mathematics Series, No. 1790, Springer-Verlag, Berlin. 